\title{
Análisis de parámetros fisicoquímicos, compuestos fenólicos y capacidad antioxidante en piel, pulpa y fruto entero de cinco cultivares de manzana (Malus domestica) cosechadas en México
}

\author{
Analysis of physicochemical parameters, phenolic compounds and antioxidant capacity of peel, pulp \\ and whole fruit of five apple varieties (Malus domestica) harvested in Mexico
}

\begin{abstract}
Lizbeth Sandra Corona Leo', Diana Maylet Hernández-Martínez², Ofelia Gabriela Meza-Márquez ${ }^{*}$
Instituto Politécnico Nacional. Escuela Nacional de Ciencias Biológicas-Zacatenco. Departamento de Ingeniería Bioquímica. Av. Wilfrido Massieu S/N, Esq. Cda. Miguel Stampa. Col. Unidad Profesional Adolfo López Mateos, Zacatenco. Alcaldía Gustavo A. Madero. C.P. 07738. Ciudad de México, México.

2 Instituto Politécnico Nacional, Escuela Nacional de Ciencias Biológicas-Santo Tomás. Departamento de Biofísica. Prolongación de Carpio y Plan de Ayala S/N. Col. Santo Tomás. Alcaldía Miguel Hidalgo. C.P. 11340. Ciudad de México, México.
\end{abstract}

\section{RESUMEN}

La manzana es fuente de compuestos polifenólicos con propiedades antioxidantes que tienen efectos protectores para la salud humana. El objetivo del trabajo fue determinar los sólidos solubles totales (SST), acidez total (AT), $\mathrm{pH}$, compuestos fenólicos totales (CFT), contenido total de flavonoides (CTF), contenido total de antocianinas (CTA) y capacidad antioxidante (ABTS y DPPH) en cinco variedades de manzana cultivadas en México. Los resultados indican que, en todas las variedades de manzana, los CFT, CTF, CTA, ABTS y DPPH se encuentran en mayor cantidad en la piel, seguido por el fruto entero y pulpa. En la manzana Golden delicious y en la pulpa de todos los cultivares no se detectaron antocianinas. El análisis de componentes principales (ACP) indica que los SST y AT están asociados a la pulpa y fruto entero, mientras que los CFT, CTF, CTA, ABTS y DPPH están asociados a la piel del fruto. Los CFT y CTA presentaron correlación positiva ( $r$ : 0.72-0.83) con el método DPPH en piel, mientras que el ensayo ABTS tuvo correlación positiva $(r$ : $0.75-0.89)$ con CFT y CTF en piel. Los SST, AT, pH, CFT, CTF, CTA, ABTS y DPPH varían dependiendo del cultivar, región geográfica y parte del fruto. Palabras clave: Malus domestica, compuestos fenólicos, flavonoides, antocianinas, capacidad antioxidante.

\section{ABSTRACT}

Apples are a source of polyphenolic compounds with antioxidant properties that have protective effects on human health. The objective of this work was to determine total soluble solids (SST), total acidity (AT), pH, total phenolic compounds (CFT), total flavonoid content (CTF), total anthocyanin content (CTA) and antioxidant capacity (ABTS and DPPH) of five apple varieties harvested in Mexico. Results indicate that CFT, CTF, CTA, ABTS and DPPH are distributed in the following order: peel, whole fruit, pulp. In the Golden delicious variety and in the pulp of all apple varieties no anthocyanins were detected. The ACP indicates that SST and AT are associated with pulp and whole fruit, while CFT, CTF,
CTA, ABTS and DPPH are associated with the peel of the fruit. The CFT and CTA showed a positive correlation ( $r$ : 0.72-0.83) with the DPPH method in peel, while the ABTS assay had a positive correlation ( $r$ : 0.75-0.89) with CFT and CTF in peel. The SST, AT, pH, CFT, CTF, CTA, ABTS and DPPH vary depending on the cultivar, geographic region and part of the fruit.

Keywords: Malus domestica, phenolic compounds, flavonoids, anthocyanins, antioxidant capacity.

\section{INTRODUCCIÓN}

La manzana (Malus domestica) es un fruto carnoso; el color, tamaño y sabor varía dependiendo de la variedad. Este cultivo es el cuarto producto hortícola más importante de la nutrición humana en el mundo. Cerca de 716.93 millones de toneladas de manzanas se producen en el mundo, los mayores productores son China (49\%) y Estados Unidos (7 $\%)$. México es el decimotercer productor en el mundo (USDA, 2017).

El consumo de manzana se recomienda mundialmente debido a su contenido de nutrimentos y otros compuestos como los compuestos fenólicos que proporcionan capacidad antioxidante. Los antioxidantes presentes en la manzana pueden reducir el riesgo de algunas enfermedades crónicas relacionadas con el estrés oxidativo como enfermedades cardiovasculares, neurodegenerativas, infarto cerebral, diabetes, obesidad, algunos tipos de cáncer (Bouayed et al., 2011).

Los compuestos fenólicos que se encuentran comúnmente en manzana son los ácidos hidroxibenzóicos (ácido gálico, ácido syríngico, ácido gentísico), ácido hidroxicinámico y sus derivados (ácido $p$-cumárico, ácido cafeico, ácido ferúlico, ácido clorogénico), flavonoides (quercetina, epicatequina, catequina, procianidinas) y antocianinas (cianidinas y sus glucósidos) (Tsao et al., 2005). La concentración de estos compuestos fenólicos en manzana puede depender de muchos factores como el genotipo, madurez del fruto, factores ambientales, condiciones de cultivo, enfermedades del fruto, almacenamiento y procesamiento. La concentración de los

*Autor para correspondencia: Ofelia Gabriela Meza Márquez Correo electrónico:ogmmz@yahoo.com.mx 
compuestos fenólicos también varía dependiendo de la parte del fruto (entero, piel, pulpa o semillas) (Vieira et al., 2009).

Recientemente, se ha incrementado la necesidad de cuantificar los parámetros fisicoquímicos, compuestos fenólicos y capacidad antioxidante en manzana debido a que este fruto tiene relevancia comercial y económica; además, los productores de manzana exigen cada vez mejores estándares de calidad. Aunque muchos estudios han analizado los compuestos antes mencionados en varios cultivares de manzana cosechados en diferentes países como Polonia (Wojdylo et al., 2008), Brasil (Vieira et al., 2009a), Grecia (Drogoudi y Pantelidis, 2011), Chile (Quitral et al., 2013) y China (Wang et al., 2015), a nuestro conocimiento, no se ha reportado la información sobre la capacidad antioxidante y los compuestos fenólicos en manzanas cultivadas en México.

Es de importancia analizar estos compuestos ya que la calidad y cantidad de los compuestos fenólicos, así como la capacidad antioxidante de éstos, puede variar dependiendo del cultivar y región de cultivo. Existen incontables cultivares de manzana y cultivos nuevos continúan desarrollándose e introduciéndose comercialmente (Zucoloto et al., 2015), por consiguiente, el presente estudio proporciona un aporte complementario sobre el contenido y/o variación de estos compuestos en diferentes cultivares de manzana cosechada en México.

Con base en lo anterior, el objetivo del trabajo fue determinar los parámetros fisicoquímicos (sólidos solubles totales, acidez total, pH), compuestos fenólicos totales, contenido total de flavonoides, contenido total de antocianinas y capacidad antioxidante en piel, pulpa y fruto entero en cinco variedades de manzana (Gala, Starking, Granny Smith, Red delicious y Golden delicious) cultivada en México. Con fines comparativos, también se analizaron dos variedades de manzana importada (Red delicious y Golden delicious).

\section{MATERIALES Y MÉTODOS Reactivos}

Todos los reactivos fueron de grado analítico. El reactivo de Folin-Ciocalteu, 1,1-difenil-2-picrilhidrazil (DPPH), 2,2'-azino-bis (ácido 3-etilbenzotiazolina-6-sulfónico) (ABTS), ácido 6-hidroxi-2,5,7,8-tetrametilcroman-2-carboxílico (Trolox), ácido 3,4,5-trihidroxibenzoico (ácido gálico) y catequina se adquirieron de Sigma Chemical Co. (St. Louis, MO, USA). El acetato de sodio, acetona, ácido clorhídrico, bicarbonato de sodio, carbonato de amonio, carbonato de sodio, cloruro de aluminio, cloruro de calcio, cloruro de potasio, cloruro de sodio, etanol, hidróxido de sodio, metanol y persulfato de potasio se adquirieron de J.T. Baker (Center Valley, PA, USA).

\section{Muestras}

Los frutos de manzana (Malus domestica) cultivados en México se cosecharon en 2018 en el Estado de Chihuahua, México $\left(28^{\circ} 38^{\prime} 7^{\prime \prime} \mathrm{N}, 106^{\circ} 5^{\prime} 20^{\prime \prime} \mathrm{W}\right)$. Los frutos se adquirieron en madurez comercial óptimo. Las cinco variedades de manzana cultivadas en México fueron las siguientes: Gala, Starking, Granny Smith, Red delicious y Golden delicious.
Con fines comparativos se adquirieron dos variedades de manzana (Red delicious y Golden delicious) importada de California, Estados Unidos ( $35^{\circ} 07^{\prime} 32^{\prime \prime} \mathrm{N}, 117^{\circ} 59^{\prime} 09^{\prime \prime} \mathrm{W}$ ) y cosechada en 2018. Todos los frutos fueron seleccionados sin daño físico o biológico aparente, se lavaron con jabón y agua corriente y se dejaron secar a temperatura ambiente $\left(20^{\circ} \mathrm{C} \pm\right.$ $2{ }^{\circ} \mathrm{C}$ ). Posteriormente, para cada variedad, treinta manzanas se cortaron rápidamente en pequeñas rebanadas de tres maneras: 1) fruto entero (pulpa con piel y sin semillas); 2) pulpa (pulpa sin piel y sin semillas); y 3) piel de las manzanas. A cada muestra se le asignó un código para identificar la variedad de manzana y la parte analizada (piel, pulpa, fruto entero).

Las muestras de cada variedad (piel, pulpa y fruto entero) se guardaron en bolsas de polietileno selladas al vacío (Selovac, modelo 200B, Brazil), y se almacenaron en un congelador a $-20{ }^{\circ} \mathrm{C}$ hasta el análisis. Todos los análisis se llevaron a cabo en los siguientes dos meses.

\section{Análisis de parámetros fisicoquímicos}

El análisis de los parámetros fisicoquímicos se realizó en el fruto fresco (sin congelar). Los sólidos solubles totales (SST) se determinaron con base en la Norma Oficial Mexicana NMX-FF-061-2003. Los resultados se expresaron como porcentaje de SST (\% SST). La acidez total (AT) se determinó mediante la metodología reportada en la NMX-F-045-1982. Los resultados se expresaron como g de ácido málico por 100 $\mathrm{g}$ de muestra. El pH se determinó con base en la metodología indicada por Zucoloto et al. (2015). Los resultados se reportaron en escala numérica.

\section{Extracción de compuestos fenólicos}

La extracción de compuestos fenólicos se llevó a cabo con base en lo descrito por Zucoloto et al. (2015) con menores modificaciones. Aproximadamente, $5.0 \mathrm{~g}$ de muestra congelada (piel, pulpa o fruto entero) se molió en un mortero y se extrajo con $100 \mathrm{~mL}$ de metanol (70 \% v/v) por $30 \mathrm{~min}$. Posteriormente, el homogeneizado se centrifugó (ColeParmer, Mod. 5804, Vernon Hills, IL, USA.) dos veces por 10 min a $4000 \mathrm{~g}$. El sobrenadante se recolectó y se utilizó para determinar los compuestos fenólicos, flavonoides y antocianinas.

\section{Determinación de compuestos fenólicos totales}

El contenido de compuestos fenólicos totales (CFT) se determinó usando el ensayo colorimétrico de Folin-Ciocalteu descrito por Singleton et al. (1999). La absorbancia se midió a $765 \mathrm{~nm}$ contra un blanco de metanol. Los resultados se expresaron como mg equivalentes de ácido gálico (EAG) por $100 \mathrm{~g}$ de peso fresco (mg EAG/100 g PF).

\section{Determinación de flavonoides}

El contenido total de flavonoides (CTF) se llevó a cabo de acuerdo con el método colorimétrico descrito por Jia et al. (1999). La absorbancia se midió a $510 \mathrm{~nm}$ contra un blanco de metanol. Los resultados se expresaron como mg equivalentes de catequina (EC) por $100 \mathrm{~g}$ de peso fresco $(\mathrm{mg}$ $\mathrm{EC} / 100 \mathrm{~g}$ PF) 


\section{Determinación de antocianinas}

El contenido total de antocianinas (CTA) se determinó con el método diferencial de $\mathrm{pH}$ descrito por Giusti y Wrolstad (2001). Los resultados se expresaron como mg equivalentes de cianidina 3-glucósido (EC3-G) por $100 \mathrm{~g}$ de peso fresco (mg EC3-G/100 g PF).

\section{Capacidad antioxidante}

La capacidad antioxidante se determinó mediante dos metodologías: radical ABTS (2,2'-azino-bis-ácido 3-etilbenzotiazolina-6-sulfónico) (Re et al., 1999), y radical DPPH (1,1-difenil-2-picrilhidrazil) (Brand-Williams et al., 1995). Los resultados se expresaron como micromoles equivalentes de Trolox por $100 \mathrm{~g}$ de peso fresco ( $\mu$ moles ET/100 g PF).

\section{Análisis estadístico}

Todos los análisis se realizaron por triplicado. A los valores obtenidos de SST, AT, pH, CFT, CTF, CTA, ABTS y DPPH se les aplicó estadística descriptiva (media y desviación estándar), análisis de varianza (ANDEVA) de una vía y comparación múltiple de medias de Tukey $(p \leq 0.05)$. Asimismo, se realizó un Análisis de Componentes Principales (ACP) para conocer la relación entre las variables. También, se realizó un análisis de correlación de Pearson ( $r$ ) para conocer la fuerza de correlación entre las variables de respuesta. Para el análisis se utilizó el software estadístico Minitab versión 16.1.0 (State College, PA, USA).

\section{RESULTADOS Y DISCUSIÓN}

\section{Parámetros fisicoquímicos}

Los resultados de SST, AT y pH de los siete cultivares de manzana se presentan en la Figura 1. Los resultados de los parámetros fisicoquímicos demuestran que existe diferencia estadística ( $p<0.05$ ) en piel, pulpa y fruto entero. Los SST representan el contenido de azúcares, en manzana el principal azúcar es la fructosa. En la pulpa de todas las variedades de manzana se presentó el mayor porcentaje de SST debido a que en esta parte del fruto se encuentra la mayor cantidad de azúcares. El contenido de SST en pulpa varió de $9.20 \%$ en RDnPu a $13.50 \%$ en GaPu. En fruto entero, los SST fueron de $7.70 \%$ en GDnFe a $12.30 \%$ en GaFe. Por último, en piel se cuantificó el menor porcentaje de SST, éste varió de $5.20 \%$ en GDnPi a $9.90 \%$ en StPi (Figura 1A).

La norma mexicana NMX-FF-061-2003 indica que el contenido de SST en pulpa debe ser mayor de $11 \%$ en manzanas con tonalidades rojas, mixtas o parcialmente rojas, lo anterior se cumple en las variedades Gala (GaPu: $13.50 \%$ ), Starking (StPu: $12.90 \%$ ) y Red delicious importada (RDiPu: $11.10 \%)$, sin embargo, la variedad Red Delicious nacional tiene un porcentaje de SST menor (RDnPu: $9.20 \%)$ a lo establecido por la normatividad. En manzanas amarillas y verdes, el contenido de SST debe ser mayor de $12 \%$ (NMXFF-061-2003), sin embargo, en Granny Smith (GSPu: 9.90 \%), Golden delicious nacional (GDnPu: $9.90 \%$ ) y Golden delicious importada (GDiPu: $10.10 \%$ ) el porcentaje de SST es menor, lo anterior posiblemente se debe a que los frutos se cosecharon

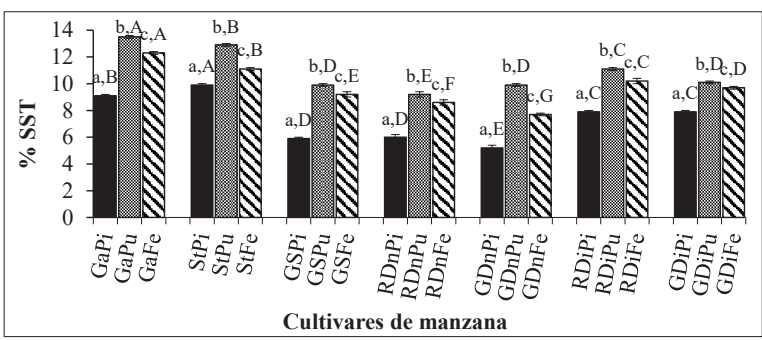

B)

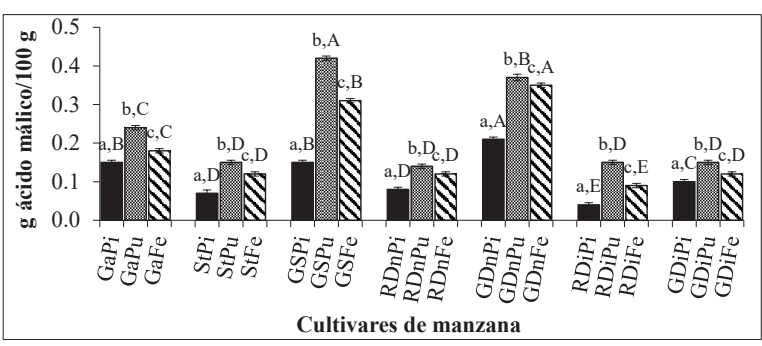

C)

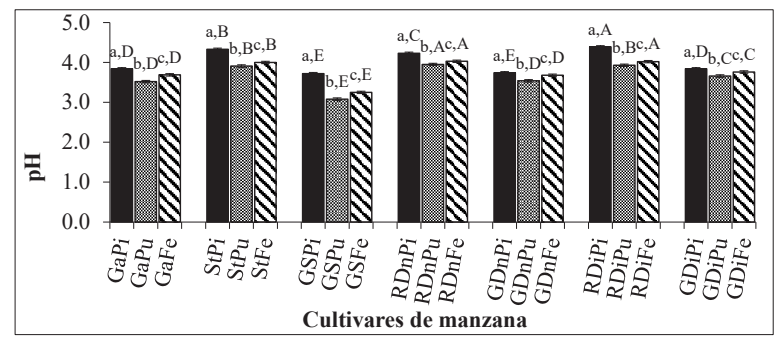

Figura 1. Parámetros fisicoquímicos de siete cultivares de manzana. A) sólidos solubles totales, B) acidez total, C) pH. Los datos representan medias \pm desviación estándar. El código está formado por la variedad (Ga: Gala, St: Starking, GS: Granny Smith, RDn:Red Delicious nacional, GDn: Golden Delicious nacional, RDi: Red Delicious importada, GDi: Golden Delicious importada) y por la parte del fruto (Pi: piel, Pu: pulpa, Fe: fruto entero). Letras minúsculas diferentes indican diferencia estadística (prueba de Tukey, $\mathrm{p} \leq$ 0.05 ) en piel, pulpa y fruto entero en el mismo cultivar. Letras mayúsculas diferentes indican diferencia estadística (prueba de Tukey, $p \leq 0.05$ ) entre los cultivares considerando la misma parte de la manzana (piel, pulpa o fruto entero).

Figure 1. Physicochemical parameters of seven apple cultivars. A) total soluble solids, B) total acidity, C) pH. Data represent means \pm standard deviation. The code consists of the variety (Ga: Gala, St: Starking, GS: Granny Smith, RDn: National Red Delicious, GDn: National Golden Delicious, RDi: Imported Red Delicious, GDi: Imported Golden Delicious) and the fruit part (Pi, peel; Pu: pulp, Fe: whole fruit). Different lowercase letters indicate statistical difference (Tukey test, $p \leq 0.05$ ) in peel, pulp and whole fruit of the same cultivar. Different capital letters indicate statistical difference (Tukey test, $p \leq 0.05$ ) between cultivars considering the same part of the apple (peel, pulp or whole fruit).

antes de tiempo o por cambios durante el manejo pos cosecha (pre enfriamiento, conservación a bajas temperaturas o atmósferas modificadas), lo que genera la disminución de los azúcares en el fruto (Melgarejo, 2010).

Drogoudi y Panteldis (2011) reportan valores de SST mayores al $12 \%$ en la pulpa de variedades de manzana roja cultivada en Grecia, estos resultados coinciden con lo reportado en el presente trabajo para la variedad Gala y Starking, sin embargo, para Granny Smith reportan un valor de $11.3 \%$, que es 1.1 veces mayor a lo reportado en el presente trabajo. Por otro lado, Drogoudi et al. (2008) reportan para Golden 
delicious el $15 \%$ de SST que es 1.5 veces mayor a los valores obtenidos en el presente trabajo para Golden delicious nacional (9.90 \%) e importada (10.10\%).

Las diferencias en el contenido de SST con respecto a otros autores probablemente se deben a las condiciones ambientales durante la producción del fruto (temperatura, luz solar e irrigación). Vieira et al. (2009a) reportaron que los SST (11.8-14.0 \%) en variedades rojas de Brasil varían significativamente e indican que el genotipo afecta la composición química de los frutos.

La acidez total (AT) está dada por los ácidos orgánicos presentes en la manzana. El ácido orgánico más importante en la manzana es el málico, aunque también se encuentran otros como el succínico. Tanto la acidez como su relación con el contenido en azúcares son componentes esenciales en la calidad de las manzanas. En la pulpa de todas las variedades de manzana se presentó el mayor contenido de AT, éste varió de $0.14 \mathrm{~g}$ ácido málico/100 g en RDnPu a $0.42 \mathrm{~g}$ ácido málico/100 g en GSPu. En fruto entero, la AT fue de $0.09 \mathrm{~g}$ ácido málico/100 g en RDiFe a $0.35 \mathrm{~g}$ ácido málico/100 g en GDnFe. Finalmente, en piel se presentó el menor contenido de AT, este valor varió de $0.04 \mathrm{~g}$ ácido málico/100 g en RDiPi a 0.21 $\mathrm{g}$ ácido málico/100 g en GDnPi (Figura 1B).

La norma mexicana NMX-F-045-1982 establece que el contenido de AT en manzana debe ser mayor a $0.30 \mathrm{y}$ menor a $0.60 \mathrm{~g}$ ácido málico/100 g. Los valores bajos de AT obtenidos en el presente trabajo podrían deberse a que la manzana es un fruto climatérico y durante su maduración los ácidos orgánicos (como el málico, succínico y cítrico) son convertidos en azúcares, disminuyendo el contenido de acidez. Asimismo, la diferencia en la concentración de ácidos orgánicos depende directamente de la maduración, de las características metabólicas del fruto y/o de la conservación frigorífica prolongada (Melgarejo, 2010).

Drogoudi y Pantelidis (2011) reportan valores de AT de 0.25 y $0.71 \mathrm{~g}$ ácido málico $/ 100 \mathrm{~g}$ en pulpa de las variedades Red delicious y Granny Smith respectivamente, estos resultados son 1.5 veces mayor a lo reportado en el presente trabajo, sin embargo, Wu et al. (2007) reportaron para Golden delicious un valor de $0.38 \mathrm{~g}$ ácido málico/100 g, este resultado es similar al obtenido en el presente trabajo para Golden delicious nacional (GDnPu: $0.37 \mathrm{~g}$ ácido málico $/ 100 \mathrm{~g}$ ).

El balance entre SST y AT permite entender las características organolépticas de las manzanas. Con base en el contenido de SST y AT, la variedad Gala podría clasificarse como agridulce, ya que la pulpa contiene más ácidos orgánicos que las otras variedades rojas, pero menos que la variedad Granny Smith.

El pH se utiliza para conocer el contenido de acidez en la manzana. En la piel de todas las variedades de manzana se presentó el mayor valor de pH, este varió de 3.72 en GSPi a 4.39 en RDiPi. En fruto entero, el pH varió de 3.25 en GSFe a 4.03 en RDnFe. Por último, en pulpa se encontró el menor valor de pH, éste varió de 3.08 en GSPu a 3.95 en RDnPu (Figura 1C).
La norma mexicana NMX-F-045-1982 establece que el $\mathrm{pH}$ debe ser mayor a 3.5 y menor a 4.0. Las variaciones en el valor de $\mathrm{pH}$ probablemente se deben a la maduración del fruto. La concentración de ácidos orgánicos en el fruto del manzano disminuye durante la maduración lo que da como consecuencia el aumento en el pH. A su vez, el pH condiciona la actividad de muchas enzimas responsables del ablandamiento, el color u otros cambios asociados con la maduración (Melgarejo, 2010).

Los valores de $\mathrm{pH}$ reportados para manzana entera coinciden con los reportados por Vieira et al. (2009a) para variedades rojas cultivadas en Brasil ( $\mathrm{pH}$ 3.9-4.27) y por $\mathrm{Wu}$ et al. (2007) para las variedades Golden Delicious (pH 3.59) y Granny Smith (pH 4.16) cultivadas en China.

La AT y el pH están relacionados ya que al haber mayor cantidad de ácidos orgánicos el pH disminuye. En la pulpa de las variedades mexicanas Granny Smith y Golden delicious se observa mayor acidez total, por lo tanto, el pH de estas variedades es menor y el sabor es más ácido. Wu et al. (2007) reportaron valores altos de $\mathrm{pH}$ (4.15) y valores bajos de AT $(0.28 \%)$ y de SST (10.48 \%) para la variedad Red delicious cultivada en China. Resultados similares se obtuvieron en el presente trabajo para la variedad Red delicious nacional e importada.

\section{Compuestos bioactivos}

Los resultados del contenido de CFT, CTF y CTA de los siete cultivares de manzana se presentan en la Figura 2. Los resultados de los compuestos bioactivos demuestran que existe diferencia estadística $(p<0.05)$ en piel, pulpa y fruto entero. El mayor contenido de CFT se presentó en la piel de todas las variedades de manzana, le sigue el fruto entero y pulpa. En la piel, el contenido de CFT varió de 209.2 a 447.87 mg EAG/100 g PF en GDiPi y RDnPi respectivamente. En fruto entero, el contenido de CFT fue de $137.2 \mathrm{mg} \mathrm{EAG/100} \mathrm{g} \mathrm{PF}$ en GDiFe a $315.87 \mathrm{mg}$ EAG/100 g PF en GSFe. En pulpa se cuantificó el menor contenido de CFT, éste varió de 109.20 a $257.20 \mathrm{mg}$ EAG/100 g PF en GDiPu y GSPu respectivamente (Figura 2A).

Las tres variedades de manzana de color rojo (Gala, Starking, Red delicious) cultivadas en México tienen mayor cantidad de CFT en comparación con la variedad Red delicious importada, incluso la variedad Granny Smith cuya piel es de color verde tiene mayor cantidad de CFT que las variedades importadas (Red y Golden delicious). La variedad Golden delicious cultivada en México tiene mayor cantidad de CFT en comparación con la variedad importada. Estas diferencias pueden deberse a varios factores como la región de cultivo, condiciones ambientales durante la producción, época de cosecha y madurez del fruto (Drogoudi et al., 2008).

Vieira et al. (2011) reportaron para Golden delicious un contenido de CFT en piel (304.66 \pm 3.74$)$ y pulpa (128.33 $\pm 4.51 \mathrm{mg} \mathrm{EAG} / 100 \mathrm{~g} \mathrm{PF}$ ), estos resultados son mayores a los reportados en el presente trabajo para la misma variedad. Bouayed et al. (2011) reportó un contenido de CFT en 
A)

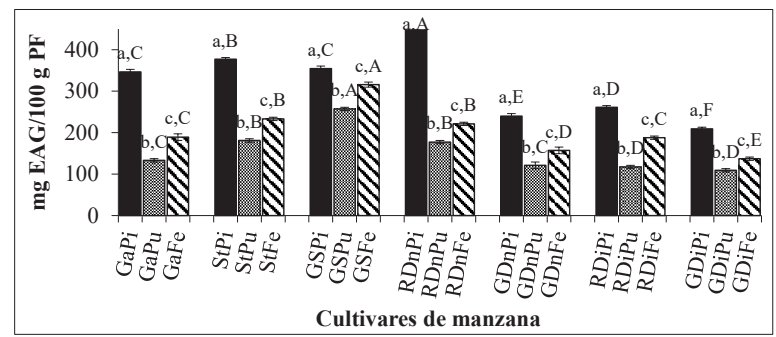

B)

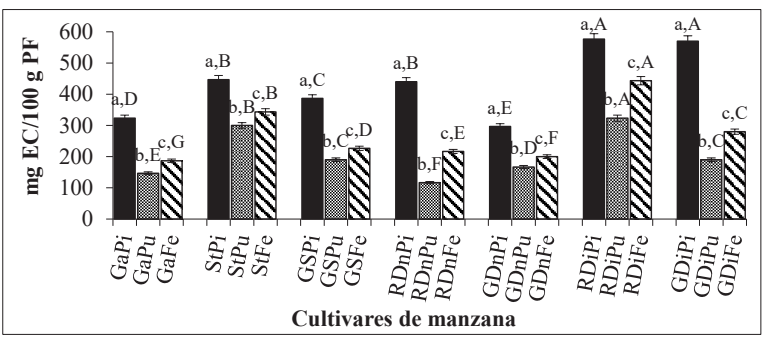

C)

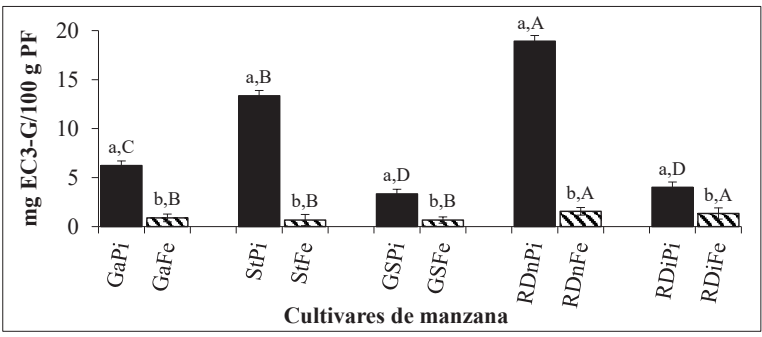

Figura 2. Compuestos bioactivos de siete cultivares de manzana. A) compuestos fenólicos totales, B) contenido total de flavonoides, C) contenido total de antocianinas. Los datos representan medias \pm desviación estándar. Códigos como la Figura 1. Letras minúsculas diferentes indican diferencia estadística (prueba de Tukey, $\mathrm{p} \leq 0.05$ ) en piel, pulpa y fruto entero en el mismo cultivar. Letras mayúsculas diferentes indican diferencia estadística (prueba de Tukey, $\mathrm{p} \leq 0.05$ ) entre los cultivares considerando la misma parte de la manzana (piel, pulpa o fruto entero).

Figure 2. Bioactive compounds of seven apple cultivars. A) total phenolic content, B) total flavonoids content, C) total anthocyanins content. Data represent means \pm standard deviation. Codes as in Figure 1. Different lowercase letters indicate statistical difference (Tukey test, $p \leq 0.05$ ) in peel, pulp and whole fruit of the same cultivar. Different capital letters indicate statistical difference (Tukey test, $p \leq 0.05$ ) between cultivars considering the same part of the apple (peel, pulp or whole fruit).

fruto entero de $110 \mathrm{mg}$ EAG/100 g PF para Golden delicious cultivada en Europa, este valor es menor al reportado en el presente trabajo para la misma variedad.

Drogoudi y Pantelidis (2011) reportaron en pulpa de Granny Smith y Red delicious concentraciones de CFT de 31.2 y $83.5 \mathrm{mg} E A G / 100 \mathrm{~g} \mathrm{PF}$, respectivamente, valores muy inferiores a los reportados en el presente trabajo para las mismas variedades (GSPu: $257.20 \mathrm{mg} \mathrm{EAG/100} \mathrm{g} \mathrm{PF,} \mathrm{RDnPu:}$ $177.20 \mathrm{mg} \mathrm{EAG} / 100 \mathrm{~g}$ PF y RDiPu: $117.20 \mathrm{mg}$ EAG/100 g PF). Quitral et al. (2013) reportaron en fruto entero de Granny Smith y Gala cultivadas en Chile un contenido de CFT de 142.72 y $130 \mathrm{mg}$ EAG/100 g PF respectivamente, estos valores son menores a los reportados en el presente trabajo para las mismas variedades (315.87 y $189.2 \mathrm{mg} \mathrm{EAG/100} \mathrm{g} \mathrm{PF,}$ respectivamente). Wang et al. (2015) reportaron concentraciones de CFT para la piel de Gala (164.18 mg EAG/100 g PF) y Golden delicious (289.94 mg EAG/100 g PF), y para pulpa
(16.03 y 28.54 mg EAG/100 g respectivamente). Comparando estos resultados con los reportados en el presente trabajo, la variedad Gala en piel y pulpa tiene respectivamente 2.1 y 8 veces más CFT. En cuanto a la manzana Golden delicious, los resultados son similares en piel, sin embargo, en pulpa, la manzana Golden delicious cultivada en México es 4.2 veces mayor en CFT.

El contenido de CFT de las manzanas cultivadas en México se comparan favorablemente con lo reportado por otros autores en otras partes del mundo. En general, los resultados muestran que los compuestos fenólicos varían considerablemente de acuerdo con la variedad de manzana, la parte del fruto analizado y el lugar de cultivo ya que el análisis estadístico presentó diferencia significativa $(p<0.05)$ en los resultados obtenidos. Incluso se ha reportado que las variaciones en el contenido fenólico pueden deberse al método de extracción (Vieira et al., 2009b; Quitral et al., 2013; Drogoudi y Pantelidis, 2011 y Wang et al., 2015).

Por otro lado, el mayor contenido total de flavonoides (CTF) se presentó en la piel de todas las variedades de manzana, le sigue el fruto entero y pulpa. En la piel el CTF fue de 296.67 a 576.67 mg EC/100 g PF en GDnPi y RDiPi respectivamente. En fruto entero, el CTF fue de $186.67 \mathrm{mg} \mathrm{EC/100}$ g PF en GaFe a 443.33 mg EC/100 g PF en RDiFe. En pulpa se cuantificó el menor CTF, éste varió de 116.67 a $323.33 \mathrm{mg}$ $\mathrm{EC} / 100 \mathrm{~g}$ PF en RDnPu y RDiPu respectivamente (Figura 2B). En la piel de la manzana se cuantificó el mayor CTF y esto se debe a que los flavonoides presentes en la piel del fruto lo protegen del daño producido por agentes oxidantes, además, éstos funcionan como protectores contra la luz UV o contra la infección por organismos fitopatógenos.

De las variedades cultivadas en México, Starking tiene mayor CTF en piel (446.67 mg EC/100 g PF), pulpa (300.00 $\mathrm{mg} \mathrm{EC} / 100 \mathrm{~g} \mathrm{PF}$ ) y fruto entero (343.33 mg EC/100 g PF) en comparación con las otras variedades. Las variedades Red delicious y Golden delicious importadas tienen mayor CTF en piel (576.67 y $570 \mathrm{mg} \mathrm{EC/100} \mathrm{g} \mathrm{PF} \mathrm{respectivamente)} \mathrm{en}$ comparación con las mismas variedades nacionales (440 y $296.67 \mathrm{mg} \mathrm{EC} / 100 \mathrm{~g} \mathrm{PF}$ respectivamente).

Los flavonoides son los compuestos fenólicos mayoritariamente presentes en manzana (Figura 2B). Debido a que los flavonoides son parte de los compuestos fenólicos, esto podría indicar que el método Folin-Ciocalteu para cuantificar fenoles totales sobreestima los resultados (Tsao, 2003). Resultados similares se han reportado en otros estudios (MooHuchin et al., 2015).

Wolfe et al. (2003) reportaron para la variedad Golden delicious concentraciones de flavonoides en piel $(167.4 \mathrm{mg}$ $\mathrm{EC} / 100 \mathrm{~g} \mathrm{PF}$ ), fruto entero (61 mg EC/100 g PF) y pulpa (42.5 $\mathrm{mg} \mathrm{EC} / 100 \mathrm{~g} \mathrm{PF}$ ), estos valores representan entre 2 y 4 veces menos que lo obtenido en el presente trabajo para la misma variedad. Asimismo, los autores reportaron para las variedades rojas Rome Beauty e Idared concentraciones de flavonoides en piel (306.1 y $303.2 \mathrm{mg} \mathrm{EC/100} \mathrm{g} \mathrm{PF} \mathrm{respectivamente),}$ fruto entero (77.1 y $55.8 \mathrm{mg} \mathrm{EC} / 100 \mathrm{~g}$ PF respectivamente) y pulpa (46.8 y $35.7 \mathrm{mg} \mathrm{EC/100} \mathrm{g} \mathrm{PF} \mathrm{respectivamente).} \mathrm{Aunque}$ 
estas variedades de manzana no se analizaron en el presente trabajo, sirven para demostrar que las variedades rojas mexicanas se comparan favorablemente con otras variedades rojas internacionales. Bouayed et al. (2011) reportaron para Golden delicious concentraciones de flavonoides en fruto

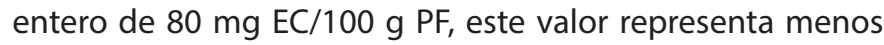
de la mitad del contenido de flavonoides encontrado en la misma variedad mexicana.

El CTF depende de muchos factores como el tipo de cultivar, estaciones de crecimiento y prácticas agrícolas. Boyer y Hair (2004) encontraron que la fertilización del árbol de manzano con nitrógeno se asoció con la disminución en antocianinas y flavonoides; por el contrario, la fertilización con calcio se asoció con el aumento en antocianinas y flavonoides.

El contenido total de antocianinas (CTA) se presenta en la Figura 2C. Estos compuestos sólo se cuantificaron en piel y fruto entero de las variedades rojas (Gala, Starking, Red delicious nacional e importada) y Granny Smith. En la variedad Golden delicious nacional e importada no se cuantificaron antocianinas. En la piel se cuantificó el mayor CTA, éste varió de 3.34 a 18.92 mg EC3-G/100 g PF en GSPi y RDnPi respectivamente. En fruto entero se cuantificó el menor CTA (0.67 a 1.56 mg EC3-G/100 g PF en GSFe y RDnFe respectivamente). En pulpa no se cuantificó antocianinas, lo anterior coincide con lo reportado por otros autores. Vieira et al. (2009b) reportaron que la pulpa de las variedades Fuji y Epagri (ambas de tonalidades rojizas) no contiene antocianinas; Vieira et al. (2011) tampoco cuantificaron antocianinas en Golden delicious.

La piel de la variedad Red delicious nacional (RDnPi) tiene mayor CTA (18.92 mg EC3-G/100 g PF) en comparación con la misma variedad importada (RDiPi: 4.01 mg EC3-G/100 g PF). Lo anterior podría indicar que la manzana red delicious cultivada en México es mejor que la importada en lo que se refiere a contenido de antocianinas, las cuales son potentes antioxidantes. El color de la manzana puede variar dependiendo de la cantidad y composición de antocianinas presentes en la piel del fruto. El principal compuesto responsable del color en manzana roja es cianidina 3-glucósido (Kalinowska et al.,2014). Las diferencias en las concentraciones de antocianinas en manzana generalmente se atribuyen al cultivar, genotipo, región de cultivo o condiciones climáticas (baja temperatura durante la noche o alta exposición al sol durante la maduración) (Wojdylo et al., 2008; Vieira et al., 2009b).

Las antocianinas son pigmentos que abarcan desde el color rojo hasta el azul, por tal motivo, estos compuestos generalmente se cuantifican en la piel de las variedades rojas, excluyendo las manzanas con tonalidades verdes y amarillas. Sin embargo, en la variedad Granny Smith se cuantificaron antocianinas en piel (3.34 mg EC-3G/100 g) y fruto entero (0.67 mg EC-3G/100 g), lo anterior probablemente se debió a que estos frutos fueron sobreexpuestos a la radiación solar. Drogoudi y Pantelidis (2011) indican que las manzanas sobreexpuestas al sol pueden desarrollar una coloración rojiza (también llamada ruborización) que propicia la síntesis de antocianinas. Asimismo, Fouché et al. (2010) señalan que en la variedad Granny Smith, la síntesis de antocianinas se da en respuesta a la alta incidencia de radiación solar.

La piel de las manzanas se desecha frecuentemente durante la elaboración de productos, subproductos o antes de comer, sin embargo, la piel es la parte que contiene el mayor contenido de fenoles, flavonoides y antocianinas en comparación con la pulpa y el fruto entero por lo que es recomendable ingerir el fruto sin eliminar la piel de éste para obtener la mayor cantidad de estos compuestos bioactivos benéficos para la salud. Otros autores describieron previamente resultados similares (Wolfe et al., 2003; Drogoudi et al., 2008).

\section{Capacidad antioxidante}

Los resultados de capacidad antioxidante mediante los ensayos ABTS y DPPH se presentan en la Figura 3. Los resultados presentan diferencia estadística $(p<0.05)$ en piel, pulpa y fruto entero. La mayor capacidad antioxidante por el ensayo ABTS se registró en la piel de todas las variedades de manzana, le sigue el fruto entero y pulpa. En piel, la capacidad antioxidante varió de 2545.45 a 4133.71 umoles ET/100 g PF en GSPi y RDnPi, respectivamente. En fruto entero, la capacidad antioxidante fue de 2016.01 a 2879.62 umoles ET/100 g PF en GSFe y GDiFe respectivamente. Por último, en la pulpa se encontró la menor capacidad antioxidante en GDiPu y RDiPu (1367.80 a 2559.08 umoles ET/100 g PF, respectivamente) (Figura $3 \mathrm{~A})$.

La mayor capacidad antioxidante mediante el ensayo DPPH se encontró en la piel de todas las variedades de manzana, le sigue el fruto entero y pulpa. En piel, la capacidad antioxidante varió de 1507.46 a 2243.01 rmoles ET/100 g PF en GDnPi y RDnPi respectivamente. En fruto entero, la capacidad antioxidante fue de 924.21 a 1729.73 umoles ET/100 g PF en GDiFe y RDiFe respectivamente. En la pulpa se registró la menor capacidad antioxidante en GSPu y RDnPu (811.57 a 1417.32 umoles ET/100 g PF, respectivamente) (Figura 3B).

En la piel de todas las variedades se registró la mayor capacidad antioxidante (por ambos ensayos) y esto se debe a que en esta parte del fruto se encuentra gran cantidad de compuestos fenólicos como flavonoides y antocianinas que ayudan a proteger el fruto de agentes agresores externos (plagas, enfermedades, pesticidas y radiación UV). Los resultados del presente trabajo coinciden con otros autores (Drogoudi et al., 2008; Drogoudi y Pantelidis, 2011).

El ensayo ABTS registró mayor capacidad antioxidante que el ensayo DPPH y esto se debe a que cada método de análisis tiene diferentes mecanismos de acción. El ensayo ABTS se basa en la generación del radical ABTS+ que es aplicable a los sistemas antioxidantes hidrófilos y lipófilos mientras que el ensayo DPPH usa un radical disuelto en medios orgánicos $\mathrm{y}$, por lo tanto, se aplica a sistemas hidrofóbicos (Brand-Williams et al., 1995; Re et al., 1999). 
A)

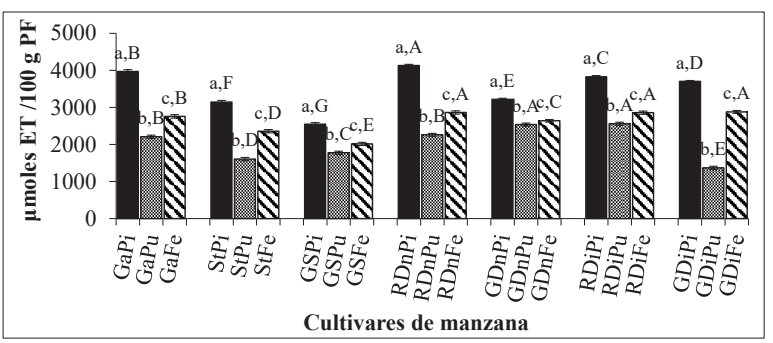

B)

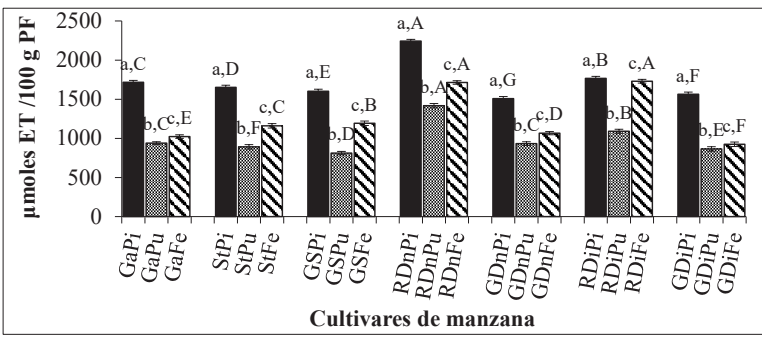

Figura 3. Capacidad antioxidante de siete cultivares de manzana. A) ensayo ABTS, B) ensayo DPPH. Los datos representan medias \pm desviación estándar. Códigos como la Figura 1. Letras minúsculas diferentes indican diferencia estadística (prueba de Tukey, $\mathrm{p} \leq 0.05$ ) en piel, pulpa y fruto entero en el mismo cultivar. Letras mayúsculas diferentes indican diferencia estadística (prueba de Tukey, $\mathrm{p} \leq 0.05$ ) entre los cultivares considerando la misma parte de la manzana (piel, pulpa o fruto entero).

Figure 3. Antioxidant Capacity of seven apple cultivars. A) ABTS assay, B) DPPH assay. Data represent means \pm standard deviation. Codes as in Figure 1. Different lowercase letters indicate statistical difference (Tukey test, $p$ $\leq 0.05$ ) in peel, pulp and whole fruit of the same cultivar. Different capital letters indicate statistical difference (Tukey test, $p \leq 0.05$ ) between cultivars, considering the same part of the apple (peel, pulp or whole fruit).

\section{Análisis de componentes principales (ACP)}

La Figura 4 presenta las cargas obtenidas con los dos primeros componentes principales (PC) mismos que explican el $76.8 \%$ de la variabilidad. La mayor parte de la variabilidad está contenida en el PC1 (59.7\%) que está relacionado positivamente con los compuestos fenólicos, antocianinas, flavonoides, ABTS y DPPH. El PC2 es responsable del $17.1 \%$ de la variabilidad y se asocia positivamente con SST y $\mathrm{pH}$, pero negativamente con AT. La AT se ubica de lado contrario al pH ya que ambos parámetros están relacionados debido a

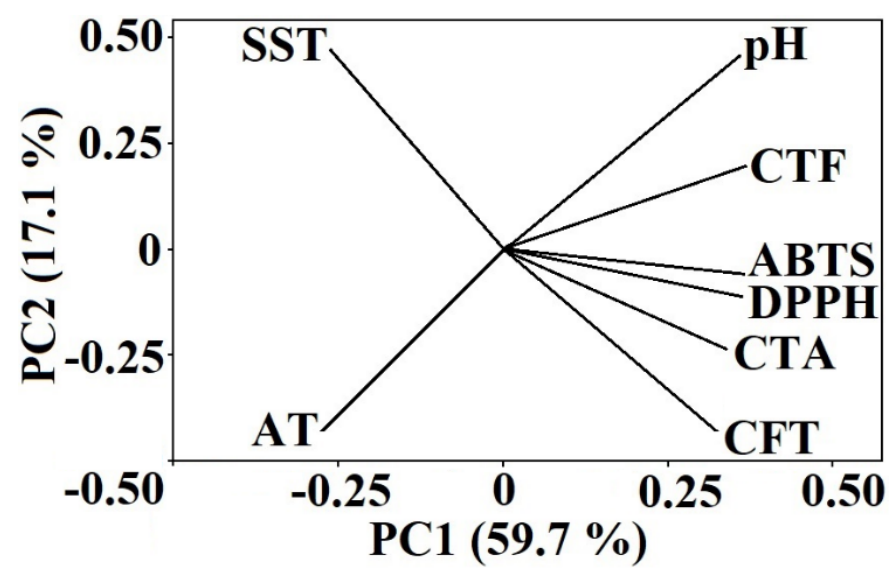

Figura 4. Gráfica de cargas del Análisis de Componentes Principales (ACP). Figure 4. Principal components loading plot. que al haber mayor (o menor) cantidad de ácidos orgánicos el pH disminuye (o aumenta). El CTF, CTA y la capacidad antioxidante (ABTS y DPPH) están relacionados de tal manera que la capacidad antioxidante de los frutos puede atribuirse a los flavonoides y antocianinas.

El ACP permitió reducir el número de variables de tal manera que las variedades de manzana y partes del fruto se correlacionan con los parámetros fisicoquímicos y compuestos bioactivos (Figura 5). Las variedades de manzana se distribuyen en tres grupos: el primer grupo (circulo de color verde) posee características similares de AT en las variedades Granny Smith (pulpa, fruto entero) y Golden delicious nacional (pulpa, fruto entero); el segundo grupo (circulo de color rojo) tiene valores similares de SST en pulpa y fruto entero de las variedades Gala, Golden delicious importada, Starking, Red delicious importada y Red delicious nacional (pulpa). Por último, el tercer grupo (circulo de color azul) tiene alto contenido de CFT, CTF, CTA y capacidad antioxidante. El fruto entero de la variedad Red delicious nacional (RDnFe) se asocia con un alto $\mathrm{pH}$, mientras que la piel de las variedades Gala, Starking, Granny Smith, Red delicious (nacional e importada) y Golden delicious (nacional e importada) se asocian con los compuestos fenólicos, flavonoides, antocianinas y capacidad antioxidante (DPPH y ABTS).

Finalmente, cabe señalar que el ACP demostró que la piel de todas las variedades de manzana tiene el mayor contenido de compuestos fenólicos, flavonoides, antocianinas y capacidad antioxidante y esto se debe a que en esta parte del fruto se encuentran compuestos que no se presentan en pulpa, por ejemplo, a parte de catequinas, epicatequinas, procianidinas (B1 y B2), ácido cafeico y ácido clorogénico, en la piel se encuentran antocianinas y glucósidos de quercetina (Tsao et al., 2005).

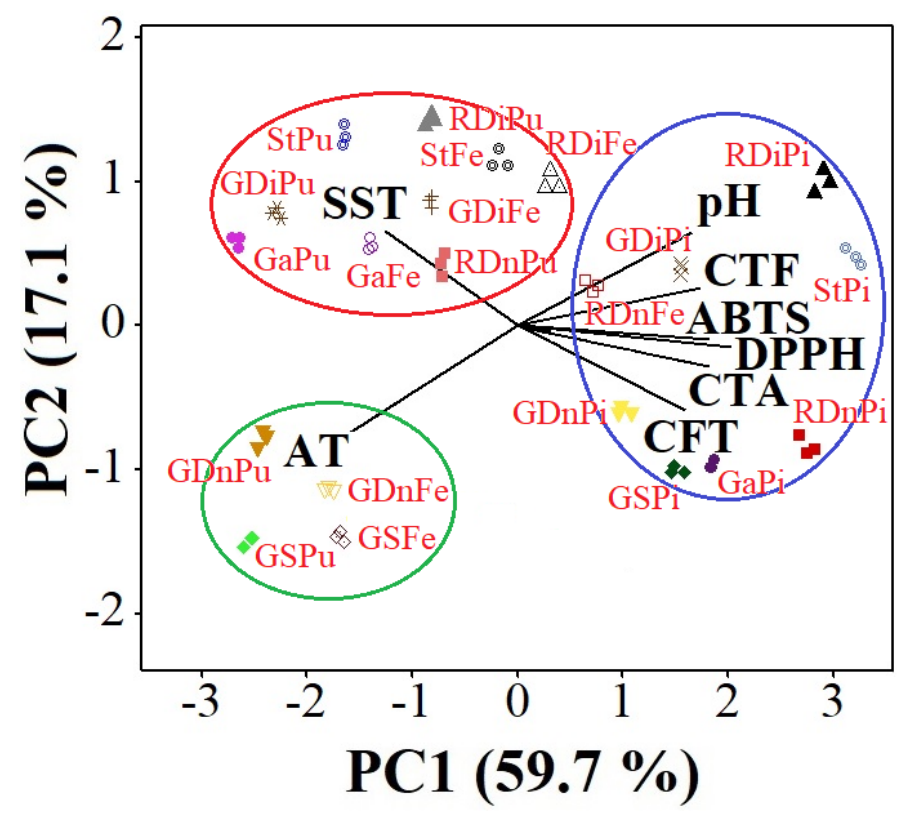

Figura 5. Gráfica Biplot del ACP de siete cultivares de manzana. Códigos como la Figura 1.

Figure 5. Biplot for the PCA of seven apple cultivars. Codes as in Figure 1. 


\section{Análisis de correlación de Pearson}

Los resultados del análisis de correlación de Pearson mostraron una asociación significativa $(p<0.01)$ entre la capacidad antioxidante y los compuestos analizados (Tabla 1). En la piel de las manzanas se presentó una correlación positiva, de moderada a grande entre los compuestos fenólicos, flavonoides y antocianinas con el método ABTS $(0.73>r<$ $0.89)$ y DPPH $(0.72>r<0.83)$. En la pulpa de las manzanas hay correlaciones positivas con los compuestos fenólicos ( $r$ $=0.69)$ y flavonoides $(r=0.81)$ con el método ABTS, mientras que con el método DPPH se presentaron correlaciones bajas en ambos compuestos $(r<0.18)$. En fruto entero se encontró una correlación positiva y grande en flavonoides con método ABTS $(r=0.87)$ y DPPH $(r=0.85)$. Los resultados indican que probablemente los flavonoides y las antocianinas contribuyen significativamente a la capacidad antioxidante en manzana. Una tendencia similar se ha reportado en otros estudios (Vieira et al., 2009b).

Tabla 1. Correlación de Pearson para los compuestos fenólicos y capacidad antioxidante de siete cultivares de manzana.

Table 1. Pearson's correlation for phenolic compounds and antioxidant capacity of seven apple cultivars.

\begin{tabular}{lccc}
\hline Muestra & Compuesto & ABTS & DPPH \\
\hline \multirow{3}{*}{ Piel } & CFT & 0.75 & 0.72 \\
& CTF & 0.89 & 0.74 \\
& CTA & 0.73 & 0.83 \\
Pulpa & CFT & 0.69 & 0.10 \\
& CTF & 0.81 & 0.18 \\
\multirow{3}{*}{ Fruto entero } & CFT & 0.63 & 0.21 \\
& CTF & 0.87 & 0.85 \\
\hline
\end{tabular}

\section{CONCLUSIONES}

La composición fisicoquímica (SST, AT, pH) y contenido de compuestos bioactivos (compuestos fenólicos, flavonoides, antocianinas) en manzana (Malus domestica) presenta diferencia estadística $(p<0.05)$ debido a varios factores, entre ellos el tipo de cultivar, región geográfica y parte del fruto. Las variedades rojas (Red delicious > Starking > Gala) presentaron mayor contenido de compuestos fenólicos, flavonoides, y en particular antocianinas. El contenido de compuestos fenólicos, flavonoides, antocianinas y capacidad antioxidante en todos los cultivares de manzanas fue alta en la piel en comparación con la pulpa y el fruto entero, esto indica que la piel de la manzana son una mejor fuente de compuestos fenólicos que la pulpa y el fruto entero. Con base en el ACP, la piel de todas las variedades de manzana se asocia a los compuestos fenólicos, flavonoides, antocianinas y capacidad antioxidante. El análisis de correlación de Pearson demostró que la capacidad antioxidante se correlacionó con flavonoides y antocianinas. Con base en los resultados, las manzanas cultivadas en México se comparan con aquéllas cultivadas en otras regiones y pueden ser excelente fuente de compuestos fenólicos. Los resultados obtenidos en el pre- sente trabajo ayudarían a seleccionar las mejores variedades de manzana para su comercialización, así como implementar estrategias para el mejoramiento de los cultivos con el fin de obtener genotipos con alta calidad nutricional y compuestos fenólicos.

\section{AGRADECIMIENTOS}

Los autores desean agradecer al Consejo Nacional de Ciencia y Tecnología (CONACyT) y Secretaría de Investigación y Posgrado del Instituto Politécnico Nacional (SIP-IPN).

\section{REFERENCIAS}

Bouayed, J., Hoffmann, L. y Bohn, T. 2011. Total phenolics, flavonoids, anthocyanins and antioxidant activity following simulated gastro-intestinal digestion and dialysis of apple varieties: Bioaccessibility and potential uptake. Food Chemistry. 128: 14-21.

Boyer, J. y Hai, R. 2004. Apple phytochemicals and their health benefits. Nutrition Journal. 3: 1-15.

Brand-Williams, W., Cuvelier, M.E. y Berset, C. 1995. Use of free radical method to evaluate antioxidant activity. LWT-Food Technology. 28: 25-30.

Drogoudi, P.D. y Pantelidis, G. 2011. Effects of position on canopy and harvest time on fruit physico-chemical and antioxidant properties in different apple cultivars. Scientia Horticulturae. 129: 752-760.

Drogoudi, P.D, Michailidis, Z. y Pantelidis, G. 2008. Peel and flesh antioxidant content and harvest quality characteristics of seven apple cultivars. Scientia Horticulturae. 115: 149-153.

Fouché, J., Roberts, S., Midgley, J.E. y Steyn, W. 2010. Peel color and blemishes in 'Granny Smith' apples in relation to canopy light environment. Scientia Horticulturae. 45: 899-905.

Giusti, M., y Wrolstad, R.E. 2001. Characterization and measurement of anthocyanins by UV-visible spectroscopy. Current Protocols in Food Analytical Chemistry. F1.2.1-F1.2.13.

Jia, Z., Mengcheng, T. y Jianming, W. 1999. Determination of flavonoid contents in mulberry and their scavenging effects on superoxide radicals. Food Chemistry. 64: 555-559.

Kalinowska, M., Bielawska, A., Lewandowska-Siwkiewicz H., Priebe W., y Lewandowski, W. 2014. Apples: content of phenolic compounds vs. variety, part of apple and cultivation model, extraction of phenolic compounds, biological properties. Plant Physiology and Biochemistry. 84: 169-188.

Melgarejo, L.M. 2010. Experimentos en fisiología vegetal. Charlie's impresores Ltda. Bogotá, Colombia.

Moo-Huchin, V.M., Moo-Huchin, M.I., Estrada-León, R.J., CuevasGlory, L., Estrada-Mota, I.A., Ortiz-Vazquez, E., BetancurAncona, D. y Sauri-Duch, E. 2015. Antioxidant compounds, antioxidant activity and phenolic content in peel from three tropical fruits from Yucatan, México. Food Chemistry. 166: 17-22.

Norma Oficial Mexicana NMX-F-045-1982. Alimentos. Frutas y derivados. Jugo de manzana. [Consultado Julio 2019]. 1982. Disponible en: https://www.colpos.mx/bancodenormas/ nmexicanas/NMX-F-045-1982.PDF

Norma Oficial Mexicana NMX-FF-061-2003, productos agrícolas no industrializados para consume humano-fruta fresca- 
manzana (Malus pumila Mill). (Malus domestica Borkh)Especificaciones. [Consultado Julio 2019]. 2003. Disponible en: https://www.colpos.mx/bancodenormas/nmexicanas/ NMX-FF-061-2003.PDF

Quitral, V., Sepúlveda, M. y Schwartz, M. 2013. Antioxidant capacity and total polyphenol content in different apple varieties cultivated in Chile. Revista Iberoamericana de Tecnología Postcosecha. 14: 31-39.

Re, R., Pellegrini, N., Proteggente, A., Pannala, A., Yang, M. y RiceEvans, C. 1999. Antioxidant activity applying an improved ABTS radical cation decolorization assay. Free Radical Biology and Medicine. 26: 1231-1237.

Singleton, V.L., Orthofer, R. y Lamuela-Raventós, R.M. 1999. Analysis of total phenols and other oxidation substrates and antioxidants by means of Folin-Ciocalteu reagent. Methods in Enzymology. 299: 152-178.

Tsao, R., Yang, R., Young, J.C. y Zhu, H. 2003. Polyphenolic profiles in eight apple cultivars using High-Performance Liquid Chromatography (HPLC). Journal of Agricultural and Food Chemistry. 51: 6347-6353.

Tsao, R., Yang, R., Xie, S., Sockovie, E. y Khanizadeh, S. 2005. Which polyphenolic compounds contribute to the total antioxidant activities of apple?. Journal of Agricultural and Food Chemistry. 53: 4989-4995.

USDA. 2017. Agricultural Statistics 2017. United States Government. Washington, D.C.

Vieira, F.G.K., Borges, G.S.C., Copetti, C., Amboni, R.D.M.C., Denardi, F.y Fett, R. 2009a. Physico-chemical and antioxidant properties of six apple cultivars (Malus domestica Borkh) grown in southern Brazil. Scientia Horticulturae. 122: 421425.
Vieira, F.G.K., Borges, G.S.C., Copetti, C., Gonzaga, L.V., Costa, E. y Fett, R. 2009b. Activity and contents of polyphenolic antioxidants in the whole fruit, flesh and peel of three apple cultivars. Archivos Latinoamericanos de Nutrición. 59: 101105.

Vieira, F.G.K., Borges, G.S.C., Copetti, C., Pietro, P.F., Nunes, E.C. y Fett, R. 2011. Phenolic compounds and antioxidant activity of the apple flesh and peel of eleven cultivars grown in Brazil. Scientia Horticulturae. 128: 261-266.

Wang, X., Li, C., Liang, D., Zou,Y., Li, P. y Ma, F. (2015). Phenolic compounds and antioxidant activity in red-fleshed apples. Journal Functional Foods. 18: 1086-1094.

Wojdylo, A., Oszmianski, J. y Laskowski, P. 2008. Polyphenolic compounds and antioxidant activity of new and old apple varieties. Journal of Agricultural and Food Chemistry. 56: 6520-6530.

Wolfe, K., Wu, X. y Liu, R.H. 2003. Antioxidant activity of apple peels. Journal of Agricultural and Food Chemistry. 51: 609614.

Wu, J., Gao, H., Zhao, L., Liao, X., Chen, F., Wang, Z. y Hu, X. 2007. Chemical compositional characterization of some apple cultivars. Food Chemistry. 103: 88-93.

Zucoloto, M., Mosbah, K., Kushad, M. y Sawwan, J. 2015. Bioactive compounds and quality characteristics of five apples cultivars. Ciência Rural. 45: 1972-1979. 